\title{
Accelerated series for universal constants, by the WZ method
}

\author{
Herbert S. Wilf
}

Department of Mathematics, University of Pennsylvania

Philadelphia, PA 19104-6395

received June 11, 1999, revised August 30, 1999, accepted September 10, 1999.

In this paper, the author presents a method, based on WZ theory, for finding rapidly converging series for universal constants. This method is analogous but different from Amdeberhan and Zeilberger's method.

Keywords: WZ theory, series convergence, hypergeometric series

In [1] Amdeberhan and Zeilberger have given a general method, based on WZ theory, for finding rapidly converging series for universal constants. We give another, somewhat different method here. In the form that we shall give to the method, the summand will satisfy a first order homogeneous recurrence but the sum will satisfy a first order inhomogeneous recurrence. What we obtain are remarkable families of representations of the constants, one for each $n \geq 0$. If we look at eq. (5) below, for instance, we see that the constant $\pi^{2} / 6$ is equal to any one of infinitely many series, one for each $n$, one of which converges geometrically rapidly.

\section{General formulation}

Let $F(n, k)$ satisfy a first order recurrence of the form

$$
a_{0}(n) F(n, k)+a_{1}(n) F(n+1, k)=G(n, k+1)-G(n, k),
$$

where $a_{0}, a_{1}$ are polynomials, and $\lim _{k \rightarrow \infty} G(n, k)=0$ for nonnegative $n$. Define

$$
f(n)=\sum_{k \geq 0} F(n, k) \quad(n=0,1,2,3, \ldots),
$$

which is assumed to be convergent for nonnegative $n$.

By summing (1) over $k \geq 0$ we find that

$$
a_{0}(n) f(n)+a_{1}(n) f(n+1)=-G(n, 0) .
$$

1365-8050 C 1999 Maison de l'Informatique et des Mathématiques Discrètes (MIMD), Paris, France 
This can be "solved" by the usual methods. If we define

$$
A(n)=\prod_{j=0}^{n-1}\left(-\frac{a_{0}(j)}{a_{1}(j)}\right)
$$

then after the change of dependent variable $f(n)=A(n) h(n)$ in (2) it takes the form

$$
h(n+1)-h(n)=-\frac{G(n, 0)}{a_{1}(n) A(n+1)} .
$$

Thus we have

$$
h(n)=-\sum_{j=0}^{n-1} \frac{G(j, 0)}{a_{1}(j) A(j+1)}+h(0),
$$

and so

$$
f(n)=A(n)\left\{f(0)-\sum_{j=0}^{n-1} \frac{G(j, 0)}{a_{1}(j) A(j+1)}\right\}
$$

which is to say that

$$
\sum_{k \geq 0} F(n, k)=A(n)\left\{\sum_{k \geq 0} F(0, k)-\sum_{j=0}^{n-1} \frac{G(j, 0)}{a_{1}(j) A(j+1)}\right\} .
$$

We are interested in the constant

$$
C=\sum_{k \geq 0} F(0, k)
$$

so, solving for it, we have our main result which is

$$
C=\sum_{j=0}^{n-1} \frac{G(j, 0)}{a_{1}(j) A(j+1)}+\frac{1}{A(n)} \sum_{k \geq 0} F(n, k) .
$$

We note that the left side is independent of $n$, hence we have an infinite number of representations of the constant $C$, one for each $n=0,1,2, \ldots$.

Here are two examples, in one of which $C$ turns out to be $\pi^{2} / 6$ and in the other it is $\zeta(3)$.

1. Take $F(n, k)=k !^{2} /(n+k+1) !^{2}$. Then $F(0, k)=1 /(k+1)^{2}$ and therefore the constant $C=\pi^{2} / 6$. By Zeilberger's algorithm, $F$ satisfies the recurrence

$$
-(4 n+2) F(n, k)+(n+1)^{3} F(n+1, k)=G(n, k+1)-G(n, k),
$$

where $G(n, k)=(3 n+3+2 k) F(n, k)$. Thus we have $G(n, 0)=(3 n+3) /(n+1) !^{2}, a_{0}(n)=$ $-4 n-2, a_{1}(n)=(n+1)^{3}$. By (3) we find

$$
A(n)=\prod_{j=0}^{n-1}\left(\frac{4 j+2}{(j+1)^{3}}\right)=\frac{(2 n) !}{n !^{4}}
$$


Substituting all of this in (4), we have finally

$$
3 \sum_{j=1}^{n} \frac{1}{j^{2}\left(\begin{array}{c}
2 j \\
j
\end{array}\right)}+\frac{n !^{4}}{(2 n) !} \sum_{k \geq 0} \frac{k !^{2}}{(k+n+1) !^{2}}=\frac{\pi^{2}}{6}
$$

In (5), when $n=0$ we have the usual series for $\zeta(2)$, and when " $n=\infty$ " we have a much more rapidly convergent (known) representation, namely

$$
\frac{\pi^{2}}{6}=3 \sum_{j=1}^{\infty} \frac{1}{j^{2}\left(\begin{array}{c}
2 j \\
j
\end{array}\right)}
$$

But (5) holds for every $n \geq 0$. Indeed, when $n=1$ it states that

$$
\sum_{k \geq 1} \frac{1}{k^{2}(k+1)^{2}}=\frac{\pi^{2}}{3}-3 .
$$

Bill Gosper has also found (p.c.) the identity (5) by his method of "path-invariant matrix hacking."

2. Now take $F(n, k)=k !^{3} /(k+2 n+1) !^{3}$. Then Zeilberger's algorithm finds the recurrence

$$
-6(3 n+2)(3 n+1) F(n, k)-16(2 n+1)^{3}(n+1)^{5} F(n+1, k)=G(n, k+1)-G(n, k),
$$

where now

$$
G(n, 0)=\frac{(2 n+1)\left(56 n^{2}+80 n+29\right)}{2(n+1)(2 n+1) !^{3}} .
$$

We simply substitute all of this into (4) and find that for each $n=0,1,2, \ldots$, we have

$$
\sum_{j=1}^{n}(-1)^{j+1} \frac{\left(56 j^{2}-32 j+5\right)(j-1) !^{3}}{4(2 j-1)^{2}(3 j) !}+\frac{(-1)^{n} n !^{3}(2 n) !^{3}}{(3 n) !} \sum_{k \geq 0} \frac{k !^{3}}{(k+2 n+1) !^{3}}=\zeta(3)
$$

In (7), when $n=0$ we have the usual series for $\zeta(3)$, and when " $n=\infty$ " we have a much more rapidly convergent representation, namely

$$
\zeta(3)=\sum_{j=1}^{\infty}(-1)^{j+1} \frac{\left(56 j^{2}-32 j+5\right)(j-1) !^{3}}{4(2 j-1)^{2}(3 j) !} .
$$

But (7) holds for every $n \geq 0$. Indeed, when $n=1$ it states that

$$
\sum_{k \geq 1} \frac{1}{k^{3}(k+1)^{3}(k+2)^{3}}=\frac{29}{32}-\frac{3}{4} \zeta(3),
$$

and when $n=2$ it takes the form

$$
\sum_{k \geq 1} \frac{1}{((k+1)(k+2)(k+3)(k+4)(k+5))^{3}}=\frac{5}{768} \zeta(3)-\frac{10385}{98304}
$$

Although the terms of (8) vanish exponentially rapidly, roughly like $27^{-j}$, those of the series of Amdeberhan-Zeilberger [1] go to 0 even more rapidly, like $1024^{-j}$. 


\section{References}

[1] Tewodros Amdeberhan and Doron Zeilberger, Hypergeometric series acceleration via the WZ method, Electronic J. Combinat. 4 no. 2 (1997), \#R3. 